\title{
Sur les équations linéaires aux différentielles totales
}

par

W. NIKLIBORC (Lwów).

Envisageons le système des équations linéaires aux différentielles totales:

$$
d z_{i}=\sum_{k=1}^{n} a_{i k} d x_{k} \quad(i=1,2, \ldots m)
$$

On suppose, que les $a_{i k}$ sont des fonctions des variables

$$
x_{1}, x_{2} \ldots x_{n}, z_{1}, \ldots z_{m}
$$

définies dans un certain domaine $(D)$ à $m+n$ dimensions.

Le problème de Cauchy pour les équations (1) consiste dans la recherche des fonctions

$$
z_{1}\left(x_{1} \ldots x_{n}\right), \ldots z_{m}\left(x_{1} \ldots x_{n}\right)
$$

satisfaisant aux équations (1) et vérifiant d'autre part „les conditions initiales":

$$
z_{i}\left(x_{1}^{(0)} \ldots x_{n}^{(0)}\right)=z_{t}^{(0)}, \quad(i=1,2, \ldots m)
$$
$\left(x_{1}{ }^{(o)} \ldots x_{n}{ }^{(o)}, z_{1}{ }^{(o)}, \ldots z_{m}{ }^{(o)}\right)$ étant un point intérieur de $(D)$.

Si les équations (1) forment un système "complètement intégrable“, c'est-à-dire si l'on a identiquement

$\frac{\partial a_{i k}}{\partial x_{j}}+\sum_{v=1}^{m} \frac{\partial a_{i k}}{\partial z_{v}} \cdot a_{v j}=\frac{\partial a_{i j}}{\partial x_{k}}+\sum_{v=1}^{m} \frac{\partial a_{i j}}{\partial x_{v}} \cdot a_{v k}, \quad \begin{array}{r}(i=1,2 \ldots m) \\ (k, j=1,2 \ldots n)\end{array}$ on peut, sous certaines conditions de régularité des $a_{i k}$, démontrer, qu'une solution du problème existe.

La démonstration directe de cette proposition n'était donnée à ce que je sais, que dans les $\operatorname{cas}^{1}$ ) où les fonctions $a_{i k}$ sont holomorphes dans le voisinage du point $\left.\left(x_{k}^{(o)}, z_{i}^{(0)}\right)^{2}\right)$.

1) V. p. e. Goursat. "Cours d'Analyse“. T. II. I. Éd. p. 357-360, ou les "Leçons sur l'intégration" des équations aux dérivées partielles du premier ordre ${ }^{4}$ du même Auteur. Il. Éd. p. 105-107.

2) Pour abréger nous désignos par $\left(x_{k}, z_{i}\right)$ le point $\left(x_{1} \ldots x_{n}, z_{1} \ldots z_{m}\right)$. 
Lorsqu'on considére des fonctions $\alpha_{i k}$ non analytiques, on peut démontrer la possibilité du problème, dont il s'agit, indin rectement, à savoir par la reduction au certain système des équations différentielles ordinaires ${ }^{3}$ ). Cette reduction exige certaines suppositions sur les $a_{i k}$. M. Bieberbach suppose par exemple ${ }^{4}$ ), que les fonctions $a_{i k}$ possédent des dérivées partielles de quatre premiers ordres. Il dit $^{5}$ ), il est vrai, qu'on peut diminuer ces hypothèses, mais comme il nous semble, il est nécessaire pour la validité de ce mode de démonstration de supposer, que les $a_{i k}$ possédent des dérivées partielles continues de deux premiers ordres.

Dans ce qui va suivre, nous allons prouver le théorème de Cauchy pour le système (1) d'une manière directe, en utilisant le procédé des approximations succesives.

$\S 1$. Notations et hypothèses. Envisageons un domaine $(D)$ fermé, situé dans l'espace des variables

$$
x_{1} \ldots x_{n}, z_{1}, \ldots z_{m} \text {, }
$$

et soient les $a_{i k}$ des fonctions de ces variables. Supposons, que:

10. Les fonctions $a_{i k}$ sont continues dans $(D)$, et possédent des dérivées partielles du premier ordre continues dans $(D)$ fermé.

$2^{0}$. Dans tout $(D)$ on a

$(1,1) \frac{\partial a_{i k}}{\partial x_{j}}+\sum_{v=1}^{m} \frac{\partial a_{i k}}{\partial z_{v}} \cdot a_{v j}=\frac{\partial a_{i j}}{\partial x_{k}}+\sum_{v=1}^{m} \frac{\partial a_{i j}}{\partial x_{v}} a_{r, k} \quad(i=1,2, \ldots m)$

Les fonctions $\left|a_{i, k}\right|$ étant bornées dans $(D)$, désignons par $M$ la plus petite commune borne supérieure des ces fonctions. De même soit $K$ la plus petite borne supérieure des fonctions $\left|\frac{\partial \alpha_{i k}}{\partial z_{j}}\right|$.

Pour simplifier l'écriture supposons, que les conditions initiales $\left(x_{k}{ }^{(o)}, z_{i}{ }^{(o)}\right)$ coïncident avec l'origine des coordonnées, qui, d'apres l'hypothèse, est un point intérieur de $(D)$. Ils existent alors deux nombres $a>0$ et $b>0$, tels, que le parallélepipède:

est situé dans $(D)$.

$$
\begin{array}{ll}
\left|x_{k}\right| \leqq a & (k=1,2, \ldots n) \\
z_{i} \mid \leqq b & (i=1,2 \ldots m),
\end{array}
$$

V. p. e. Goursat 1

4) "Differentialgleichungen". Springer, Berlin, 1923, p. 222.
§2. Problème et théorème. Envisageons maintenant le problème suivant:

"Déterminer $1^{0}$ un nombre positif $\delta$

$2^{0}$ un système des fonctions

$z_{1}\left(x_{1} \ldots x_{n}\right), \ldots z_{m}\left(x_{1} \ldots x_{n}\right)$

définies dans le domaine

(2)

continues dan $\left|x_{k}\right| \leq \delta \quad(k=1,2 \ldots n)$, tielles du premier ormaine avec les dérivées par-

a) vérifiant les équations (1)

b) satisfaisant aux conditions

$$
\begin{aligned}
& z_{i}(00 \ldots 0)=0 \\
& (i=1,2 \ldots m)^{\alpha} .
\end{aligned}
$$

Nous allons dèmontrer le THÉORÈME:

"Sous les conditions du $\S^{\text {-phe }}$ précédent il existe toujours une solution de la question proposée. Pour $\delta$ on peut prendre le plus petit de deux nombres $a$ et $\frac{b}{n M}$ ".

$\S 3$. Construction des approximations. Nous allons définir une suite infinie des systèmes à $m$ fonctions

dites "approximations succesives".

$$
z_{1}^{(r)}\left(x_{1} \ldots x_{n}\right), \ldots z_{m}^{(r)}\left(x_{1} \ldots x_{n}\right), \quad(r=0,1,2 \ldots)
$$

Posons

$$
\text { (4) } \begin{array}{r}
z_{i}^{(r+1)}\left(x_{1} \ldots x_{n}\right)=\int_{0}^{1} \sum_{k=1}^{n} a_{t k}\left[t x_{1}, \ldots t x_{n}, z_{1}^{(r)}\left(t x_{1} \ldots t x_{n}\right), \ldots\right. \\
\left.\ldots z_{m}^{(r)}\left(t x_{1} \ldots t x_{n}\right)\right] . x_{k} d t \quad \begin{array}{r}
(i=1,2 \ldots m) \\
(r=0,1,2 \ldots) .
\end{array}
\end{array}
$$

L'induction permet à démontrer facilement, que nos conventions définis sent les $z_{i}^{(r)}$ dans le domaine $\left.(2)^{\theta}\right)$ comme des fonctions continues, qui possédent des dérivées partielles $d u$ premier ordre, continues.

ø) Nous choisissons naturellement 8 égal à $\operatorname{Min}\left(a, \frac{b}{n M}\right)$. 
En effet, notre proposition est vraie pour le prẻmier système de notre suite. Supposons, qu'elle est vraie pour un certain $r_{2}$ et qu' on a de plus

$$
\left|z_{i}^{(r)}\left(x_{1} \ldots x_{n}\right)\right| \leq b
$$

dans tout le domaine (2). Alors on voit par les formules (4), que les $z_{i}{ }^{(r+1)}$ sont continues, possédent des dérivées partielles du premier ordre et - finalement - que

$\left|z_{i}^{(r+1}\left(x_{1} \ldots x_{n}\right)\right| \leqq \int_{0}^{1} \sum_{k=1}^{n}\left|a_{i k}\right| \cdot\left|x_{k}\right| d t \leqq n M . \delta \leqq n M \cdot \frac{b}{n M}=b$.

Notı e lemme est donc établi. Remarquons que

$$
z_{i}^{(r)}(0, \ldots 0)=0 \quad(i=1,2 \ldots m),(r=0,1,2 \ldots) .
$$

$\S 4$. Convergence des approximations succesives. Posons pour abrèger

(5) $a_{i, k}^{(r)}=a_{i, k}^{(r)}\left[x_{1} \ldots x_{n}, z_{1}{ }^{(r)}\left(x_{1} \ldots x_{n}\right), \ldots z_{m}{ }^{(r)}\left(x_{1} \ldots x_{n}\right)\right]$

et

$a_{i, k}^{(r)}(t)=a_{i, k}^{(r)}\left[t x_{1} \ldots t x_{n}, z_{1}^{(r)}\left(t x_{1} \ldots t x_{n}\right), \ldots z_{m}^{(r)}\left(t x_{1} \ldots t x_{n}\right)\right]$. On a

(6)

$$
\begin{gathered}
\left|z_{i}^{(r+1)}\left(x_{1} \ldots x_{n}\right)-z_{i}^{(r)}\left(x_{1} \ldots x_{n}\right)\right|= \\
=\left|\int_{0}^{1} \sum_{k=1}^{n}\left\{a_{i, k}(r)(t)-a_{i, k}^{(r-1)}(t)\right\} x_{k} d t\right| \leq \\
\leqq \sum_{k=1}^{n}\left|x_{k}\right| \cdot \int_{0}^{1}\left|a_{i, k}^{(r)}(t)-a_{i, k}^{(r-1)}(t)\right| d t \leqq \\
\leqq K \sum_{k=1}^{n} \sum_{l=1}^{m}\left|x_{k}\right| \cdot \int_{0}^{1}\left|z_{l}^{(r)}\left(t x_{1} \ldots t x_{n}\right)-z_{l}^{(r-1)}\left(t x_{1} \ldots t x_{n}\right)\right| d t .
\end{gathered}
$$

Supposons, que

(7)

$$
\left|\boldsymbol{z}_{i}^{(r)}-z_{i}^{(r-1)}\right| \leq \frac{M}{m K} \frac{\left\{m K \sum_{s=1}^{n}\left|x_{s}\right|\right\}^{r}}{r !}
$$

il suit de (6), que

$$
\begin{aligned}
\left|z_{i}^{(r+1)}-z_{i}^{(r)}\right| \leqq K & \sum_{k=1}^{n} \sum_{l=1}^{m}\left|x_{k}\right| \cdot \int_{0}^{1} \frac{M}{m K} \frac{\left\{m K \cdot \sum_{s=1}^{n}\left|t x_{s}\right|\right\}^{r}}{r !} d t= \\
& =\frac{M}{m K} \cdot \frac{\left\{m K \cdot \sum_{s=1}^{n}\left|x_{s}\right|\right\}^{r+1}}{(r+1) !}
\end{aligned}
$$

\section{D'autre part on a}

$$
\left|z_{i}^{(1)}-z_{i}^{(0)}\right|=\left|z_{i}^{(1)}\right| \leqq \sum_{k=1}^{n}\left|x_{k}\right| \int_{0}^{1}\left|a_{i k}^{(0)}\right| d t \leqq M . \sum_{s=0}^{n}\left|x_{s}\right| \text {. }
$$

L'inégalité (12) est alors valable dans tout le domaine (2) et pour tout $r$ naturel. La série

$$
\sum_{r=1}^{\infty}\left|z_{i}^{(r)}-z_{\mathrm{i}}^{(r-1)}\right|
$$

étant majorée par la série

$$
\sum_{r=1}^{\infty} \frac{\left\{m K \cdot \sum_{s=1}^{n}\left|x_{s}\right|\right\}}{r !}
$$

[dont la somme est $\frac{M}{m K}\left(e^{m K \sum_{s=1}^{n}\left|x_{s}\right|}-1\right)$ ], converge dans (2) uniformément. Par conséquent la suite des systèmes $z_{i}{ }^{(r)}$ converge aussi uniformément dans le domaine (2).

Désignons par

$$
z_{1}\left(x_{1} \ldots x_{n}\right), \ldots z_{m}\left(x_{1} \ldots x_{n}\right)
$$

la limite de cette suite. Les fonctions $z_{i}$ sont évidemiment continues dans le domaine (2) et on a

$$
z_{i}(00 \ldots 0)=0 \text {. }
$$

\$ 5. Calcul des dérivées des approximations. Les formules (4) fournissent:

$$
\begin{aligned}
& \frac{\partial z_{i}^{(r+1)}}{\partial x_{l}}=\int_{0}^{1}\left\{a_{i, l}^{(r)}(t)+\sum_{s=1}^{n} \frac{\partial a_{i s}{ }^{(r)}}{\partial x_{l}} t x_{s}+\right. \\
& \left.+\sum_{s=1}^{n} \sum_{j=1}^{m} \frac{\partial a_{i, s}{ }^{(r)}}{\partial z_{j}} \cdot \frac{\partial z_{j}{ }^{(r)}}{\partial x_{l}} t x_{s}\right\} d t \quad \begin{array}{l}
(i=1,2 \ldots m) \\
(l=1,2 \ldots n) \\
(r=0,1,2, \ldots)
\end{array}
\end{aligned}
$$

Les dérivées partielles des $a_{i, k}^{(r)}$, qui figurent sous le signe de l'intégrale, doivent être calculées au point

$$
t x_{1} \ldots t x_{m}, z_{1}{ }^{(r)}\left(t x_{1} \ldots t x_{n}\right), \ldots, z_{m}{ }^{(r)}\left(t x_{1} \ldots t x_{n}\right) \text {. }
$$

Or, on vérifie sans difficulté, que l' on a

$$
\begin{gathered}
t x_{l} \frac{\partial a_{i l}(r)}{\partial x_{l}}+\sum_{j=1}^{m} \frac{\partial a_{i l}{ }^{(r)}}{\partial z_{j}} \cdot \frac{\partial z_{j}^{(r)}}{\partial x_{l}} t x_{l}=t \frac{d}{d t} a_{i l}^{(r)}(t)- \\
-\sum_{\substack{s=1 \\
s \neq l}}^{n} t x_{s} \frac{\partial a_{i l}(r)}{\partial x_{s}}-\sum_{\substack{s=1 \\
s \neq l}}^{n} \sum_{j=1}^{m} \frac{\partial a_{i l}(r)}{\partial z_{j}} \cdot \frac{\partial z_{j}^{(r)}}{\partial x_{s}} \cdot t x_{s}
\end{gathered}
$$


et par conséquent

$$
\begin{aligned}
\frac{\partial z_{i}(r+1)}{\partial x_{l}}= & \int_{0}^{1}\left\{t \frac{d}{d t} a_{i, l}(r)(t)+a_{t, l} l^{(r)}(t)+\sum_{\substack{s=1 \\
s \neq l}}^{n} t x_{s} \cdot\left[\frac{\partial a_{i,}(r)}{\partial x_{l}}-\frac{\partial \alpha_{i, l}(r)}{\partial x_{s}}\right]+\right. \\
& \left.+\sum_{\substack{s=1 \\
s \neq l}}^{n} \sum_{j=1}^{m} t x_{s}\left[\frac{\partial a_{i, s}(r)}{\partial z_{j}} \cdot \frac{\partial z_{j}}{\partial x_{l}}-\frac{\partial a_{i, l}(r)}{\partial z_{j}} \cdot \frac{\partial z_{j}(r)}{\partial x_{s}}\right]\right\} d t . \\
\text { Mais } & \int_{0}^{1} 1 \frac{d}{d t} a_{i, l}(r) d t=\left.t \quad a_{i, l}(r)\right|_{0} ^{1}-\int_{0}^{1} a_{i, l}(r)(t) d t_{y}
\end{aligned}
$$

donc d'après $(5)$

$$
\begin{aligned}
& \frac{\partial z_{i}(r+1)}{\partial x_{l}}=a_{i, l}(r)+\int_{0}^{1}\left\{\sum_{\substack{s=1 \\
s \neq l}}^{n} t x_{s} \mid \frac{\partial a_{i, s}(r)}{\partial x_{l}}-\frac{\partial a_{i, l}(r)}{\partial x_{s}}\right]+ \\
& \left.\left.+\sum_{\substack{s=1 \\
s \neq j}}^{n} \sum_{\substack{j=1 \\
s \neq 1}}^{m} t x_{s} \mid \frac{\partial a_{i, s}^{(r)}}{\partial z_{j}} \cdot \frac{\partial z_{j}^{(r)}}{\partial x_{l}}-\frac{\partial a_{i, l}(r)}{\partial z_{j}} \cdot \frac{\partial z_{j}^{(r)}}{\partial x_{s}}\right]\right\} d t
\end{aligned}
$$

D'autre part on a d'aprés $(1,1)$ :

$$
\frac{\partial a_{i, s}(r)}{\partial x_{l}}-\frac{\partial a_{i, l}(r)}{\partial x_{s}}=\sum_{j=1}^{m}\left(\frac{\partial a_{i, l}(r)}{\partial z_{j}} a_{j, s}{ }^{(r)}-\frac{\partial a_{i, s}(r)}{\partial z_{j}} \cdot a_{j, l}(r)\right)
$$

donc

$$
\text { (8) } \begin{gathered}
\frac{\partial z_{i}^{(r+1)}}{\partial x_{l}}=a_{i, l}(r)+\sum_{\substack{s=1 \\
s \neq l}}^{n} \sum_{j=1}^{m} x_{s} \cdot \int_{0}^{1}\left\{\frac{\partial a_{i, l}(r)}{\partial z_{j}} \cdot\left(a_{j, s}(r)-\frac{\partial z_{j}^{(r)}}{\partial x_{s}}\right)-\right. \\
\left.-\frac{\partial l_{i, s}^{(r)}}{\partial z_{j}}\left(a_{j, l}{ }^{(r)}-\frac{\partial z_{j}^{(r)}}{\partial x_{l}}\right)\right\} t d t .
\end{gathered}
$$

Ainsi nous avons obtenu une formule fondamentale pour ce qui va suivre.

$\S 6$. Convergence des $\frac{\partial z_{i}(r)}{\partial x_{l}}$. De la formule (8) on déduit aussitốt l'inégalité:

$$
\begin{gathered}
\left|\frac{\partial z_{i}(r+1)}{\partial x_{l}}-a_{i, l}{ }^{(r+1)}\right| \leqq\left|a_{i, l}{ }^{(r+1)}-a_{i, l}{ }^{(r)}\right|+ \\
+K \cdot \sum_{\substack{s=1 \\
s \neq l}}^{\sum_{j=1}^{m}}\left|x_{s}\right| \cdot \int_{0}^{1}\left\{\left|\frac{\partial z_{j}{ }_{j}^{(r)}}{\partial x_{l}}-a_{j, l}{ }^{(r)}\right|+\left|\frac{\partial z_{j}^{(r)}}{\partial x_{s}}-a_{J, s}{ }^{(r)}\right|\right\} t r d t . \\
(i=1,2 \ldots m) .(l-1,2 \ldots n),(r=0,1,2 \ldots) .
\end{gathered}
$$

En ajoutant ces inégalités pour tous les indices $i$ et $l$, et en écrivant $j$ au lieu de $i$ dans les deux premières sommes nous obtenons

$$
\begin{gathered}
\sum_{i=1}^{m} \sum_{l=1}^{n}\left|\frac{\partial z_{j}^{(r+1)}}{\partial x_{l}}-a_{j, l}(r+1)\right| \leqq \sum_{j=1}^{m} \sum_{l=1}^{n}\left|a_{j, l}{ }^{(r+1)}-a_{j, l}{ }^{(r)}\right|+ \\
+m K \cdot \sum_{\substack{s=1 \\
s \neq l}} \sum_{j=1}^{m} \sum_{l=1}^{n}\left|x_{s}\right| \cdot \int_{0}^{1}\left|\frac{\partial z_{j}^{(r)}}{\partial x_{l}}-a_{j, l}{ }^{(r)}\right| \cdot t d t+ \\
\quad+i n n K \cdot \sum_{\substack{s=1 \\
s \neq l}}^{n} \sum_{j=1}^{m}\left|x_{s}\right| \cdot \int_{0}^{1}\left|\frac{\partial z_{j}(r)}{\partial x_{s}}-a_{j, s}{ }^{(r)}\right| \cdot t d t,
\end{gathered}
$$

ce qui avec

$$
\begin{aligned}
& \sum_{\substack{s=1 \\
s \neq l}}^{n} \sum_{j=1}^{m} \sum_{l=1}^{n}\left|x_{s}\right| \cdot \int_{0}^{1}\left|\frac{\partial z_{j}{ }^{(r)}}{\partial x_{l}}-a_{j, l} l^{(r)}\right| t d t \leqq \\
& \equiv\left(\sum_{s=1}^{n}\left|x_{s}\right|\right) \cdot \sum_{j=1}^{m} \sum_{l=1}^{n} \int_{0}^{1}\left|\frac{\partial z_{j}^{(r)}}{\partial x_{l}}-a_{j, I}{ }^{(r)}\right| \cdot t d t, \\
& \sum_{\substack{s=1 \\
s \neq i}}^{n} \sum_{j=1}^{m}\left|x_{s}\right| \cdot \int_{0}^{1}\left|\frac{\partial z_{j}{ }^{(r)}}{\partial x_{s}}-a_{j, s}{ }^{(r)}\right| t d t \leqq \\
& \Xi\left(\sum_{s=1}^{n}\left|x_{s}\right| \cdot\right) \cdot \sum_{s=1}^{n} \sum_{j=1}^{m} \int_{0}^{1}\left|\frac{\partial z_{j}^{(r)}}{\partial x_{s}}-a_{j, s}(r)\right| t d t, \\
& \left|a_{j, l^{(r+1)}}-a_{j, l^{(r)}}\right| \leqq K \cdot \sum_{v=1}^{m}\left|z_{\nu}^{(r+1)}-z_{\nu}^{(r)}\right|
\end{aligned}
$$

donne

$$
\begin{aligned}
& \sum_{j=1}^{m} \sum_{l=1}^{n}\left|\frac{\partial z_{j}^{(r+1)}}{\partial x_{l}}-a_{j, l}{ }^{(r+1)}\right| \leqq m n K \cdot \sum_{\nu=1}^{m}\left|z_{v}^{(r+1)}-z_{v}{ }^{(r)}\right|+ \\
& \quad+2 m n \cdot K \cdot\left(\sum_{s=1}^{n}\left|x_{s}\right|\right) \cdot \sum_{l=1}^{n} \sum_{j=1}^{m} \int_{0}^{1}\left|\frac{\partial z_{j}{ }_{j}^{(r)}}{\partial x_{l}}-a_{j, l}^{(r)}\right| t d \dot{t} .
\end{aligned}
$$

En posant

$$
u^{(r+1)}=\sum_{j=1}^{m} \sum_{l=1}^{n}\left|\frac{\partial z_{j}^{(r+1)}}{\partial x_{l}}-a_{j, l}^{(r+1)}\right|
$$

on obtient en vertu de (7): 


$$
\begin{aligned}
u^{(r+1)} & \leqq M \cdot m \cdot n \frac{m^{r+1} \cdot K^{r+1} \cdot\left(\sum_{k=1}^{n}\left|x_{k}\right|\right)^{r+1}}{(r+1) !}+ \\
& +2 m n \cdot K \cdot\left(\sum_{s=1}^{n}\left|x_{s}\right|\right) \cdot \int_{0}^{1} u^{(r)} \cdot t d t ;
\end{aligned}
$$

d'où (par l'induction) il vient

$$
u^{(r)} \leq M m n \frac{\left\{3 m n K \cdot \sum_{s=1}^{n}\left|x_{s}\right|\right\}^{r}}{r !} .
$$

La série

$$
\sum_{r=0}^{\infty} u^{(r)}
$$

étant donc convergente uniformément, on conclut, que les séries

$$
\sum_{r=0}^{\infty}\left|\frac{\partial z_{j}^{(r+1)}}{\partial x_{l}}-a_{j, l}{ }^{(r+1)}\right| \quad \quad\left(\begin{array}{l}
j=1,2 \ldots m) \\
(l=1,2 \ldots n)
\end{array}\right.
$$

le sont aussi; les termes

$$
\left|\frac{\partial z_{j}^{(r+1)}}{\partial x_{l}}-a_{j, l^{(r+1)}}\right|
$$

convergent donc uniformément vers zéro, et comme les $\alpha_{j, l^{(r+1)}}$ convergent dans ces conditions uniformément vers les limites

$$
a_{j, l}\left[x_{1}, \ldots x_{n}, z_{1}\left(x_{1} \ldots x_{n}\right), \ldots z_{m}\left(x_{1} \ldots x_{n}\right)\right],
$$

nous voyons que les $\frac{\partial z_{j}^{(r+1)}}{\partial x_{l}}$ tendent aussi vers les mêmes limites. Il s'en suit que les fonctions-limites $z_{i}$ possédent des dérivées partielles du prémier ordre, et en plus que $\frac{\partial z_{i}}{\partial x_{k}}=a_{i k}$.

$\S 7$. L'unicité de la solution peut être démontré comme il suit: soit $z_{i}$ une solution quelconque du problème proposé, et les $z_{i}{ }^{(r+1)}$ soient définis par les formules (3) et (4).

On a évidemment

$z_{i}\left(x_{1} \ldots \ldots x_{n}\right)=\int_{0}^{1} \sum_{k=1}^{n} a_{i k}\left[t x_{1} \ldots t x_{n}, z_{1}\left(t x_{1} \ldots t x_{n}\right), \ldots ..\right] x_{k} d t$

et par la

$$
\begin{array}{r}
\left|z_{i}-z_{i}^{(r+1)}\right| \leq \int_{0}^{1} \sum_{k=1}^{n}\left|a_{i k}-a_{i k}^{(r)}\right| \cdot\left|x_{k}\right| d t \leqq \\
n \cdot K \cdot\left(\sum_{k=1}^{n}\left|x_{k}\right|\right) \cdot \sum_{i=1}^{n} \int_{0}^{1}\left|z_{i}-z_{i}^{(r)}\right| d t,
\end{array}
$$

donc

$$
\sum_{i=1}^{m}\left|z_{i}-z_{i}^{(r-1)}\right| \leq m n K \cdot\left(\sum_{k=1}^{n}\left|x_{k}\right|\right) \cdot \int_{0}^{1} \sum_{i=1}^{m}\left|z_{i}-z_{i}^{(r)}\right| d t,
$$

d'où, en désignant par $A$ le maximum de l'expression

$$
\sum_{i=1}^{m}\left|z_{i}-z_{i}^{(0)}\right| \text {, }
$$

on obtient (par l'induction) l'inégalité

$$
\sum_{i=1}^{m}\left|z_{i}-z_{i}^{(r)}\right| \leq A \cdot \frac{\left\{m n K \cdot \sum_{k=1}^{n}\left|x_{k}\right|\right\}^{r}}{r !}
$$

qui montre, que les approximations $z_{i}^{(r)}$, fonctions parfaitement déterminées, convergent vers la solution $z^{(i)}$, donnée à l'avance; cette solution doit donc être unique, c. q. f. d.

Remarque 1. Si $n=1$, on obtient comme cas particulier le théorème classique pour les équations différentielles ordinaires.

Remarque 2. Ajoutons, que le domaine d'existence des solutions $z_{i}$ est ici, à notre connaissance, détérminé pour la première fois. 